\title{
Self-assembled monolayer field-effect transistors based on oligo-9,9'-dioctylfluorene phosphonic acids $\uparrow$
}

\author{
Bastian Gothe, ${ }^{a}$ Tjaard de Roo, ${ }^{b}$ Johannes Will, ${ }^{c}$ Tobias Unruh, ${ }^{c}$ Stefan Mecking (iD b \\ and Marcus Halik (D) *a
}

\begin{abstract}
The use of functional oligomers of $\pi$-conjugated oligofluorenes led to a region-selective assembly of amorphous monolayers which exhibit robust lateral charge transport pathways in selfassembled monolayer field-effect transistors over long distances and even in mixed monolayers of semiconducting and insulating molecules. This oligomer concept might stimulate a new molecular design of self-assembling semiconducting materials.
\end{abstract}

The charge transport in organic thin-film transistors, as promising devices in future flexible electronics, is directly related to the orientation, to the domain size and to the efficient interaction of $\pi$-stacked organic semiconducting molecules. ${ }^{1,2}$ In order to obtain an efficient transport, no intrinsic limitation in the layer thickness of the active materials is expected, due to the fact that more than 90 percent of charge carriers are located within the first monolayer of molecules in close proximity to the dielectric interface. ${ }^{3,4}$ This led to the successful usage of tailored organic molecules with various structures, but in general composed of a site selective anchor group, a long flexible alkyl chain, and $\pi$-conjugated head groups. These molecules are able to self-assemble to defined monolayers on dedicated surfaces and thereby, the $\pi$-systems create an interacting 2D-network and serve as a conductive channel in socalled self-assembled monolayer field-effect transistors (SAMFETs). ${ }^{5,6}$ The beauty of the SAMFET approach is the high reliability and precision in solution-based active layer formation on flat and rough surfaces with full control of layer thickness (determined and limited by the lengths of the molecules), in region selectivity (caused by the surface reactive

${ }^{a}$ Organic Materials \& Devices, Institute of Polymer Materials, Department of Materials Science, University Erlangen-Nürnberg, Martensstrasse 7, 91058 Erlangen, Germany.E-mail: marcus.halik@fau.de

${ }^{b}$ Chair of Chemical Materials Science, Department of Chemistry, University of Konstanz, D-78457 Konstanz, Germany

${ }^{c}$ Crystallography and Structural Physics, Friedrich-Alexander-Universität ErlangenNürnberg, Staudtstrasse 3, 91058 Erlangen, Germany anchor group) and in variability in the choice of $\pi$-system termination enabling $\mathrm{p}$ - and n-type devices. ${ }^{7-9}$ Several different $\pi$-motifs have been investigated, with $\pi$-system shapes ranging from stick-like structures, such as chloro[11-(5"'-ethyl$2,2^{\prime}: 5^{\prime}, 2^{\prime \prime}: 5^{\prime \prime}, 2^{\prime \prime \prime}: 5^{\prime \prime \prime}, 2^{\prime \prime \prime}$-quinquethien-5-yl)undecyl]dimethyl-silane and benzothieno[3,2- $b][1]$ benzo-thiophene or perylene-3,4,9,10tetracarboxylic bisimides to spherical systems such as fullerens. ${ }^{5,6,8,10}$ By comparing the performance of such SAMmolecules in SAMFET devices with similar $\pi$-systems in thin-film transistors (TFTs), it is notable that the values for charge carrier mobility in TFTs are almost orders of magnitude larger than for self-assembled molecules in SAMFETs. ${ }^{11-14}$ In order to understand the limitations in charge transport in such 2D-assembled $\pi$-systems, it is important to understand the intermolecular interactions on a near-neighbor scale and on a larger scale as determined by the channel length of a transistor device which is typically in the range of several microns (Fig. 1). For sticklike $\pi$-systems, typically, a 2D-polycrystallinity was obtained showing sufficient $\pi \pi$-stacking at least within the crystalline domains. ${ }^{8,15,16}$ The long range transport depends on the percolation of domains over the channel area $^{16}$ and on the bridging of $2 \mathrm{D}$ lattice mismatches in orientation at grain boundaries, e.g. by filling tiny gaps with mobile molecules (Fig. 1b). ${ }^{5}$ For spherical $\pi$-systems, whose $\pi \pi$-interaction is less limited

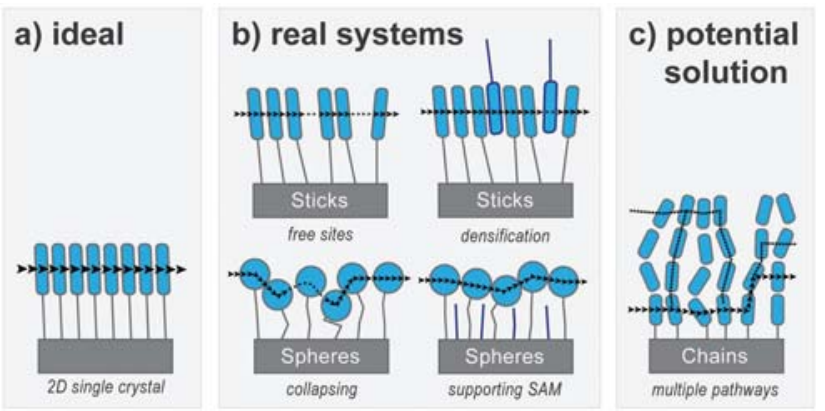

Fig. 1 Schematic self-assembled monolayer packing of semiconducting motifs and resulting charge carrier pathways. 
by lattice mismatches due to their symmetrical shape, the aspect of size mismatch on the molecular composition limits the performance. A bulky $\mathrm{C}_{60}$-head group linked on a tiny alkyl chain leads to a disordered SAM composition (Fig. 1b). It was shown that the mixed SAM deposition of functional $\mathrm{C}_{60}$-terminated molecules with supporting short alkyl-chain SAMs can improve the SAM order and thereby the charge transport properties. ${ }^{17,18}$ The common design of such SAM molecules (anchor, flexible chain and one $\pi$-system attached) implies another potential intrinsic limitation of the SAMFET concept. Even a highly polycrystalline $2 \mathrm{D}$ arrangement of one monolayer of $\pi$-systems lacks the opportunity to bypass the charge carrier across a potential mismatch within the monolayer (e.g. domain boundaries) through a second layer of the $\pi$-system on top. This missing bypass may lead to pronounced charge trapping or interruption in pathways and thereby may limit the overall device performance. Conceptually, molecules with more than one semiconductor $\pi$-system in chains would be intuitive - leading to the class of self-assembled oligomers as active components in SAMFETs (Fig. 1c).

In order to address the concept of SAMs composed of $\pi$-functional oligomers, we have employed phosphonic acid functionalized oligo-9,9'-dioctylfluorenes (OF-PA) with ten fluorene units on average $\left(M_{\mathrm{w}} / M_{\mathrm{n}}<1.2\right.$, see the ESI $\left.\dagger\right) .{ }^{19}$ These molecules exhibit covalently linked fluorene units, which have been employed as a promising p-type semi-crystalline or amorphous semiconducting polymer ${ }^{20}$ and may enable an improved percolation pathway for charge transport while forming a SAM. The key to their synthesis is controlled Suzuki-Miyaura coupling polymerization, employing a phosphonic acid (ester) functionalized palladium(II) initiator. ${ }^{19,21,22}$ We note that $\pi$-systems such as quinquethiophenes (5T) or quaterthiophenes (4T) are typically summarized as oligothiophenes, but they behave as small molecules with a defined molecular weight. Compared to the conventional small SAM molecules for SAMFET applications (sticks and spheres), the OF-PA motif differs not only in the repetition of $\pi$-systems but also in the presence of the orthogonal octyl-chains in the $9^{\prime}, 9^{\prime}$-position. The orthogonal side chain motif will likely reduce the formation of 2D crystalline domains, ${ }^{19}$ but it is strongly required to provide the solubility of the oligomer.

Phosphonic acid functionalized oligofluorenes (Fig. 2a) were assembled in a monolayer on aluminum oxide for structural studies as well as for electrical characterization in field effect transistors. The fluorene-derivative was deposited via dip coating from a toluene solution according to a process described recently. ${ }^{8,18}$ The monolayer structure was investigated by X-ray reflectivity (XRR) measurements, which provide detailed insight into the vertical molecular arrangement with sub-nanometer resolution. These structural observations are complemented with charge-transport measurements in SAMFET devices, which are typically highly sensitive to changes in the monolayer morphology. ${ }^{18}$ The combination of macroscopic measurements and structural analysis at atomicresolution enables a detailed analysis of structure-property relationships of monolayer systems.
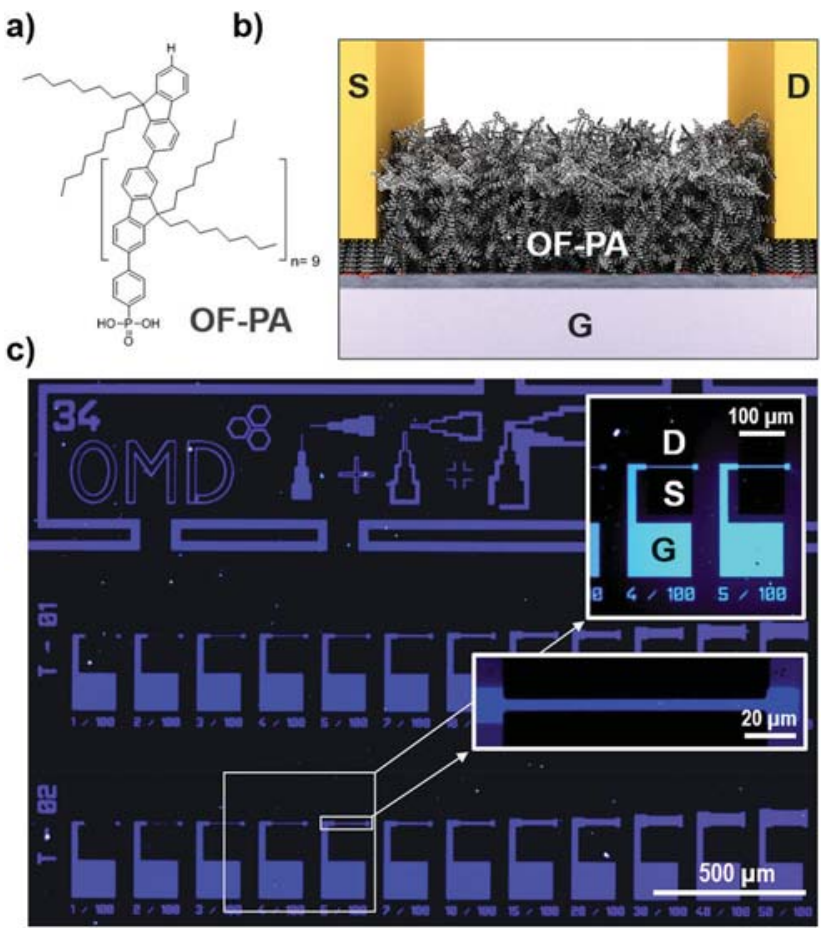

Fig. 2 Oligofluorene-phosphonic acid (OF-PA) (a) chemical structure (b) channel area schematic of a bottom-gate side-contact SAMFET (c) Fluorescence microscopy pictures of a lithographically structured chip with OF-PA SAM covered FET structures (signal gain varied with magnification). Source electrode (S), drain electrode (D) and gate electrode (G) are highlighted.

The OF-PA molecule selectively binds to the aluminum oxide (grown in an atomic layer deposition process (ALD)) driven by the phosphonic acid anchor group. It has been shown that the binding of phosphonic acid functionalized molecules follows typical Langmuir kinetics with high binding constants and results in long-term stable dense binding to oxide surfaces. ${ }^{23-25}$ The site selectivity directs the OF-PA only to the aluminum oxide covered aluminum gate electrodes. In order to prevent electrical shorts in the source-gate-overlap region of the device, these oxide areas have been coated with a thiol terminated alkyl phosphonic acid SAM (HS- $\mathrm{C}_{12}-\mathrm{PA}$ ) that acts as a dielectric layer and improves the adhesion of the gold electrodes (Fig. 2b).

Because of the oligomeric nature (high molecular weight of ca. $4044 \mathrm{~g} \mathrm{~mol}^{-1}$ compared to traditional 'small molecules') and the space requirement of the orthogonal side chains, the OF-PA is expected to create amorphous monolayers. The strong binding of the phosphonic acid anchor group ensures a close and stable packing, regardless of the oligomer size. This is indicated by static water contact angle (SCA) measurements on aluminum oxide, where the SCA changes from $72.9 \pm 0.7^{\circ}$ to $102.0 \pm 0.3^{\circ}$ after the SAM deposition. The package density of OF-PA SAMs was determined with 0.75 molecules per $\mathrm{nm}^{2}$ from thermogravimetric measurements (ESI $3 \dagger$ ) according to a reported procedure. ${ }^{25}$ Fluorescence microscopy images 
(Fig. 2c) showing large area photoemission indicate a continuous monolayer coverage and region-selective self-assembly. A band of 330 to $385 \mathrm{~nm}$ was chosen for the excitation to meet the absorption maxima determined by UV-Vis for the OF-PA (ESI $1 \dagger$ ). The absorption spectrum of the SAM corresponds to that of the corresponding amorphous polymer thin film of poly(9,9-dioctyl-9H-fluorene-2,7-diyl) (PFO) and indicates no crystallinity. $^{26}$ The fluorene emission (maximum $\left.427 \mathrm{~nm}\right)^{26}$ emanates only from the aluminum oxide covered gate pattern $(\mathrm{G})$, whereas the silicon oxide substrate areas as well as the gold source/drain electrode (S/D) areas show no emission under these measuring conditions. Strong photoluminescence from monolayer $\pi$-systems is only observed, if the incident light can couple effectively to the $\pi$-system, ${ }^{27}$ meaning not all chromophores must be oriented perpendicular to the substrate surface. ${ }^{28}$ This indicates that the fluorene chromophores are not aligned perfectly perpendicular to the surface but rather tilted or disordered and therefore exhibit this significant emission.

To investigate the molecular orientation in detail, XRR was employed as one of the most precise techniques in SAM analysis, with sub-nanometer resolution in the $z$-direction. ${ }^{29}$ The measured changes in reflection intensity are based on the electronic density differences that exist between the fluorene groups, the anchor group and the substrate in the vertical direction to the substrate normal. Fourier transformation of the XRR signal intensities gives immediate information about the layer thickness - in this case, a monolayer thickness of $3.65 \mathrm{~nm}$ for the OF-PA (Fig. 3a). In contrast to the theoretical maximum OF-PA length of $6.7 \mathrm{~nm}$ (ten monomer units), this suggests strongly tilted structures, rather than perpendicular orientation. This is reflected in the respective XRR fits. They describe a SAM layer with high roughness that increases in electron density with increasing distance from the substrate (Fig. 3b). We attribute this densification to an advancing collapse of the top repeating units because of the increase in chain flexibility while the alkyl side chains prevent tight packing at the surface. The higher roughness compared to the substrate roughness also accounts for the disordered packing (detailed fit parameters, see ESI $4 \dagger$ ). Grazing incidence measurements (GID) as well show no signal of crystalline phases, clearly indicating an amorphous structure. By combining all analytical data from XRR, GID, photoemission and TGA, the SAM of OF-PA exhibits an amorphous network with a tilted arrangement of the $\pi$-systems to the surface.

The electronic performance of SAM-based FETs is usually very sensitive to changes in the monolayer structure. ${ }^{16}$ In the following, we elucidate the electrical qualities that come with multiple $\pi$-systems. Since the monolayer molecules provide a large conjugated $\pi$-system over the backbone, charge carrier transport is possible regardless of the strong disorder as long as percolation is given between the source and the drain contact. Field-effect transistors with OF-PA as a semiconducting monolayer were successfully fabricated and electronically characterized. The monolayer assembled selectively on an aluminum oxide covered aluminum gate and was contacted with gold electrodes in a bottom contact configuration. A thiol-terminated alkyl-SAM covers the oxide areas underneath the gold electrodes (see ESI $6 \dagger$ ). This leaves the OF-PA deposition as the last process step and ensures that there is no influence on the fluorene-SAM by other process parameters. The resulting SAMFETs show p-type semiconducting behavior in high device yields of more than $95 \%$. Output and transfer curves were measured repeatedly under ambient conditions without significant degradation (Fig. 4a). The devices exhibit a hysteresis, addressed to charge trapping on free binding sites of the $\mathrm{AlO}_{x}$ surface. The extracted carrier mobilities reach a mean value of $3.6 \pm 0.3 \times 10^{-5} \mathrm{~cm}^{2} \mathrm{~V}^{-1} \mathrm{~s}^{-1}$ over 25 measured devices. These mobilities are less than one order of magnitude lower than that obtained from conventional TFTs with thicker PFO films. ${ }^{20}$ This drop in performance from TFT to SAMFET is still similar to or even less than that for thiophene-based SAMFETs compared to their conventional thin film devices, composed of quater- and quinque-thiophenes. ${ }^{5,11,30}$ In fact, other semiconductor molecules exhibit a much larger decrease up to 4 orders of magnitude from the thin film to the SAM. ${ }^{8,31}$ a)

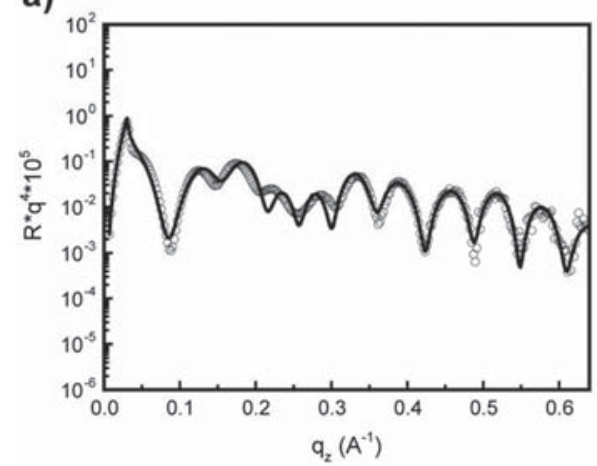

b)

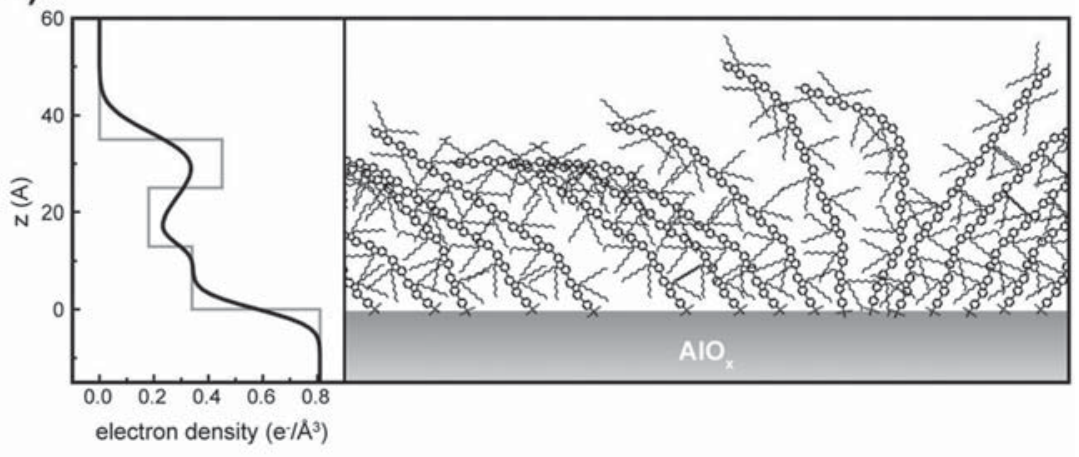

Fig. 3 X-Ray reflectivity of OF-PA SAM on $\mathrm{AlO}_{x}$ with (a) the respective fit curve. (b) Fit-derived electron density profile and schematic interpretation of tilted OF-PA with high roughness. 
a)

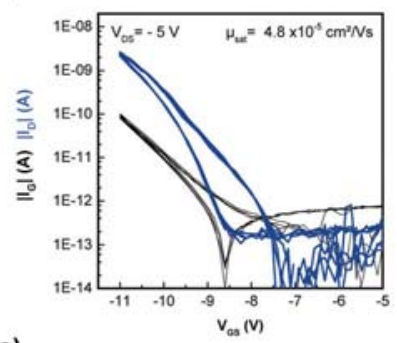

c)

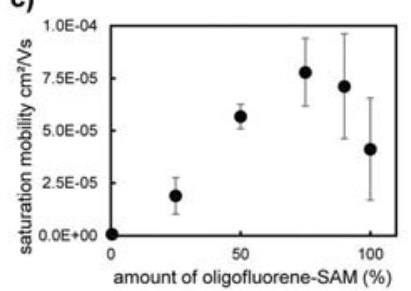

b)

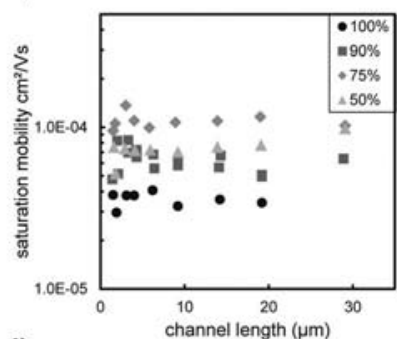

d)

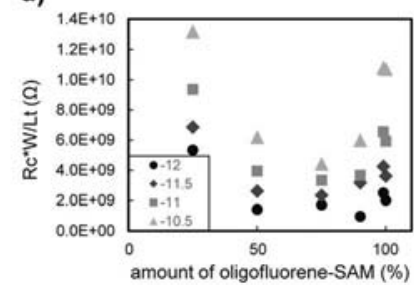

Fig. 4 (a) Representative examples of OF-PA SAMFET transfer curves. Five cycles are shown, with drain current $\left(I_{D}\right)$ in blue and gate current $\left(I_{\mathrm{G}}\right)$ in black. (b) Hole mobilities of mixed SAMFETs versus their channel lengths. (c) Mean hole mobilities at OF-PA contents of $0 \%$ to $100 \%$ in a SAM mixture with C18-PA. (d) Contact resistances of SAMFETs with the respective fluorene content. Resistance values are channel width $(W)$ and transfer length $\left(L_{t}\right)$ normalized.

In contrast to other SAMFET devices, ${ }^{5,8}$ which exhibit strong dependence of charge carrier mobility on the channel length, the OF-PA devices show virtually no dependency (Fig. 4b). These findings indicate that the concept of longer $\pi$-chains enables efficient charge transport even with a reduced molecular order of the molecules in the SAM. The benefits of the SAMFET concept (facile processing with a defined film thickness and region-selectivity and low operation voltage of transistors) are also preserved with self-assembled oligomers.

In order to investigate the impact of the SAM morphology on the charge transport, we have varied the SAM composition. The amount of OF-PA on the surface was decreased incrementally and replaced by a commercially available insulating $n$-octyl phosphonic acid $\mathrm{C}_{18}$-PA by applying the corresponding stoichiometric mixtures of the molecules. This results in randomly mixed monolayers of the two molecules on the surface with the same stoichiometry as provided by the initial solution. ${ }^{32,33}$ The changes in the morphology and electrical performance were analyzed with XRR and transistor measurements. The combination of both links the structural changes in the SAM to their effect on macroscopic properties, in this case, transistor characteristics.

SAMFETs with mixed OF-PA/ $\mathrm{C}_{18}$-PA SAMs as the active channel material were fabricated and successfully operated with OF-PA concentrations of 100, 90, 75, 50 and 25\% (Fig. 4c). Carrier mobilities peak at $75 \%$ OF-PA with a mean of $7.8 \times$ $10^{-5} \mathrm{~cm}^{2} \mathrm{~V}^{-1} \mathrm{~s}^{-1}$. With the increasing amount of $\mathrm{C}_{18}$-PA in the SAM, a reduced hysteresis in devices was obtained (ESI 7 $\dagger$ ) due to the reduced trap sites covered by the smaller $\mathrm{C}_{18}$-PA on the $\mathrm{AlO}_{x}$ surface. Remarkably, SAMFETs with only 50\% fluorene exhibit higher values than those of devices with $100 \%$ fluor-

ene. The morphology appears to be more critical to charge transport than the overall chromophore density. It is known that alkyl-phosphonic acids can help stabilizing SAMs with a strong mismatch in the space requirement of the head group and the anchor group. ${ }^{18,34}$ In the case of OF-PA, a mismatch is introduced by the orthogonal octadecyl side chains and the electrical data suggest that this mismatch is balanced best at $75 \%$ OF-PA.

Conductive pathways are still present even at low amounts $(25 \%)$ of OF-PA, which suggests that tilted oligomers maintain percolation over large distances. An additional indication for this effect is given by SCA measurements. Already at $25 \%$ OF-PA content, the SCA on the SAM is close to that of a pure OF-PA SAM (see ESI $2 \dagger$ ), meaning the surface energy is already dominated by the fluorene units.

Device performance is not only governed by the monolayer itself but also by the charge injection from the electrodes. Transmission line measurements reveal the geometry normalized contact resistance of the gold electrode/SAM-interface (Fig. 4d). ${ }^{35}$ The contact resistances of $2-11 \mathrm{k} \Omega \mathrm{cm}^{-1}$ (at $V_{\mathrm{GS}}=$ -12 ) are probably due to the erratic contact between the SAMs and the gold electrodes. Since a side-contact thin film transistor layout was used, the injection region is limited to a very small area of the devices and thus is highly sensitive to processing errors. The addition of the alkyl SAM led to a lowering of $R_{\mathrm{c}} W$ and follows the same trend in device performance improvement as obtained for the charge carrier mobility values as a function of mixing ratio.

XRR was used to study the influence of the $\mathrm{C}_{18}$-PA addition on the molecular order in the monolayer. The codeposition with $\mathrm{C}_{18}$-PA had a clear influence on the reflection curves (see ESI $5 \dagger$ ). The Fourier transforms of the XRR data reveal a decrease in the SAM thickness with lowering fluorene content in the SAM (Fig. 5a). The overall monolayer height decreased from the previously described $3.65 \mathrm{~nm}$ to $1.9 \mathrm{~nm}$ at $25 \%$ OF-PA amount. At $25 \%$, the fit-derived electron density curve (Fig. 5b) exhibits a high roughness, low electron-density layer on top of the alkyl monolayer. We attribute this layer to strongly tilted or even face-on lying fluorene chains.

Furthermore, the lowered electron density of the first SAM slab reflects the higher space requirement of the OF-PA compared to the reference $\mathrm{C}_{18}$-PA SAM. The weak features that occur around 1.2-1.4 $\mathrm{nm}$ at OF-PA concentrations of 50\% and above can be assigned to the internal order in the SAM, either from the anchor region or a densified area at the monolayer top surface. The confirmed tilt structures explain the fact that charge transport through the monolayer is still possible at very low fluorene contents. This is an inherent advantage over short SAM molecules with a very confined $\pi$-system, where the long range order is crucial for the transport. We expect that due to the long conjugated backbone, OF-PA provides multiple pathways through which charge carriers can move regardless of the overall disorder. This advantage becomes obvious at the reduced chromophore content in the SAM. 
b)

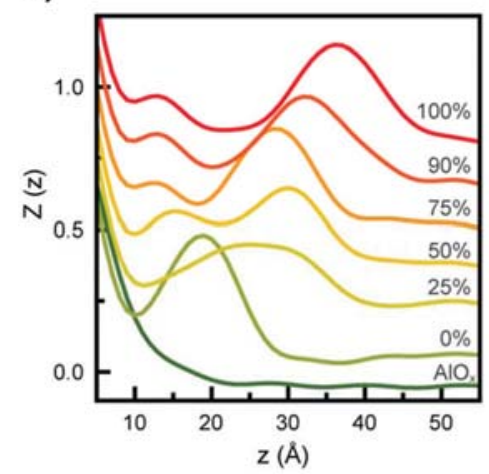

a)

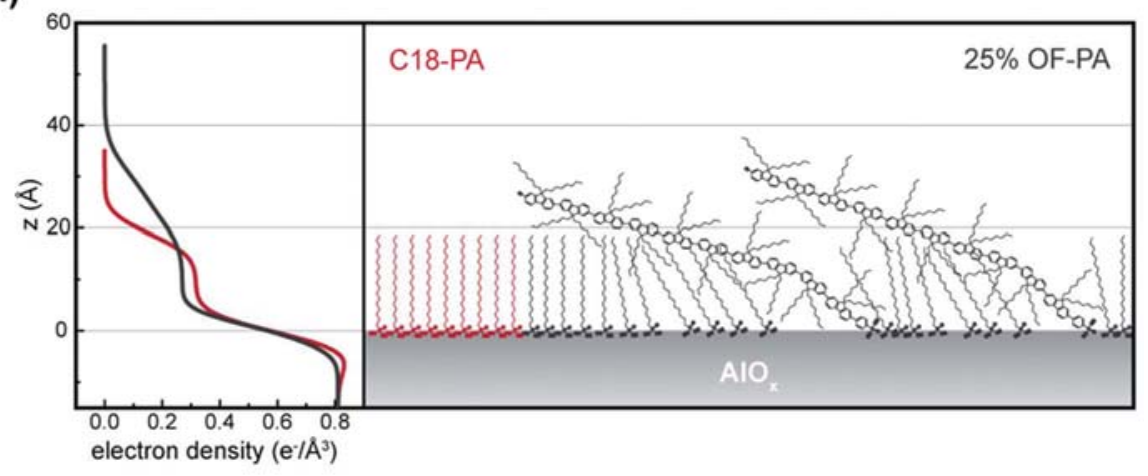

Fig. 5 (a) Fourier transform of the XRR spectra of mixed SAM layers of different compositions on AlO ${ }_{x}$ surfaces. The overall SAM-layer height decreases with increasing $\mathrm{C}_{18}$-PA amount in the SAM. (b) Fit-derived electron density curve of a mixed SAM of $25 \%$ OF-PA/75\% $\mathrm{C}_{18}$-PA and pure $\mathrm{C}_{18}$-PA layer with schematic interpretation. In the case of the mixed SAM, the alkyl layer is topped with a high-roughness fluorene layer due to the strong tilt of the oligomers.

\section{Conclusions}

We have introduced a new class of semiconducting SAMs and have discussed their morphology and charge transport properties. The concept of SAMs composed of $\pi$-functional oligomers was successfully realized for the example of phosphonic acid functionalized oligo-9',9-dioctylfluorenes (OF-PA). SAMs were assembled with strong region-selectivity on aluminum oxide surfaces. The SAMs exhibit luminescence. XRR and GID suggest a tilted and amorphous molecular arrangement. The SAMFET devices operate with excellent yields and good mobilities compared to conventional PFO devices. The transport is characterized by the enablement of multiple pathways due to the long chain structure of the OF-PA molecules and the amorphous nature of the SAM. These pathways remain operative even for long channels up to $30 \mu \mathrm{m}$ and even if the active $\pi$-component is diluted down to $25 \%$ in the channel region by co-self-assembly with insulating $\mathrm{C}_{18}$-PA. At $75 \%$ OF-PA content, the maximum device performance was obtained. This underlines the inherent advantage of oligomeric SAMs with several $\pi$-systems in the backbone which provide a robust function in devices and low sensitivity to $\pi \pi$-mismatches. We suggest planar oligomer systems as a next step, to further improve on the $\pi \pi$-stacking which is currently limited by the tetrahedral orientation of the alkyl side chains of the fluorene unit.

\section{Conflicts of interest}

There are no conflicts to declare.

\section{Acknowledgements}

The authors are grateful to acknowledge the funding of the German Research Council (DFG) within the frameworks of the Cluster of Excellence "Engineering of Advanced Materials", the
Collaborative Research Center SFB 953 "Synthetic Carbon Allotropes" and the SFB 767 "Controlled Nanosystems". The authors also thank the Erlangen Graduate School of Molecular Science (GSMS) for financial support. The GIXD experiments were performed at the European Synchrotron Radiation Facility (ESRF), Grenoble, France. We are grateful to Oleg Konovalov at the ESRF for providing assistance in using beamline ID10.

\section{References}

1 H. Sirringhaus, P. J. Brown, R. H. Friend, M. M. Nielsen, K. Bechgaard, B. M. W. Langeveld-Voss, A. J. H. Spiering, R. A. J. Janssen, E. W. Meijer, P. Herwig and D. M. de Leeuw, Nature, 1999, 401, 685-688.

2 G. Horowitz and M. E. Hajlaoui, Adv. Mater., 2000, 12, 1046-1050.

3 M. Mottaghi and G. Horowitz, Org. Electron., 2006, 7, 528-536.

4 J. J. Brondijk, W. S. C. Roelofs, S. G. J. Mathijssen, A. Shehu, T. Cramer, F. Biscarini, P. W. M. Blom and D. M. de Leeuw, Phys. Rev. Lett., 2012, 109, 56601.

5 E. C. P. Smits, S. G. J. Mathijssen, P. A. van Hal, S. Setayesh, T. C. T. Geuns, K. A. H. A. Mutsaers, E. Cantatore, H. J. Wondergem, O. Werzer, R. Resel, M. Kemerink, S. Kirchmeyer, A. M. Muzafarov, S. A. Ponomarenko, B. de Boer, P. W. M. Blom and D. M. de Leeuw, Nature, 2008, 455, 956-959.

6 M. Novak, A. Ebel, T. Meyer-Friedrichsen, A. Jedaa, B. F. Vieweg, G. Yang, K. Voitchovsky, F. Stellacci, E. Spiecker, A. Hirsch and M. Halik, Nano Lett., 2011, 11, 156-159.

7 A. Ringk, W. S. Christian Roelofs, E. C. P. Smits, C. van der Marel, I. Salzmann, A. Neuhold, G. H. Gelinck, R. Resel, D. M. de Leeuw and P. Strohriegl, Org. Electron., 2013, 14, 1297-1304.

8 T. Schmaltz, A. Y. Amin, A. Khassanov, T. MeyerFriedrichsen, H.-G. Steinrück, A. Magerl, J. J. Segura, 
K. Voitchovsky, F. Stellacci and M. Halik, Adv. Mater., 2013, 25, 4511-4514.

9 D. O. Hutchins, O. Acton, T. Weidner, N. Cernetic, J. E. Baio, G. Ting, D. G. Castner, H. Ma and A. K.-Y. Jen, Org. Electron., 2012, 13, 464-468.

10 A. Ringk, X. Li, F. Gholamrezaie, E. C. P. Smits, A. Neuhold, A. Moser, C. Van der Marel, G. H. Gelinck, R. Resel, D. M. de Leeuw and P. Strohriegl, Adv. Funct. Mater., 2013, 23, 2016-2023.

11 M. Halik, H. Klauk, U. Zschieschang, G. Schmid, S. Ponomarenko, S. Kirchmeyer and W. Weber, Adv. Mater. 2003, 15, 917-922.

12 A. Y. Amin, A. Khassanov, K. Reuter, T. Meyer-friedrichsen and M. Halik, J. Am. Chem. Soc., 2012, 134, 16548-16550.

13 C. Huang, S. Barlow and S. R. Marder, J. Org. Chem., 2011, 76, 2386-2407.

14 T. B. Singh, N. S. Sariciftci, H. Yang, L. Yang, B. Plochberger and H. Sitter, Appl. Phys. Lett., 2007, 90, 213512.

15 S. G. J. Mathijssen, E. C. P. Smits, P. A. van Hal, H. J. Wondergem, S. A. Ponomarenko, A. Moser, R. Resel, P. A. Bobbert, M. Kemerink, R. A. J. Janssen and D. M. de Leeuw, Nat. Nanotechnol., 2009, 4, 674-680.

16 T. Schmaltz, B. Gothe, A. Krause, S. Leitherer, H.-G. Steinrück, M. Thoss, T. Clark and M. Halik, ACS Nano, 2017, 11, 8747-8757.

17 C. M. Jäger, T. Schmaltz, M. Novak, A. Khassanov, A. Vorobiev, M. Hennemann, A. Krause, H. Dietrich, D. Zahn, A. Hirsch, M. Halik and T. Clark, J. Am. Chem. Soc., 2013, 135, 4893-4900.

18 T. Schmaltz, A. Khassanov, H.-G. Steinrück, A. Magerl, A. Hirsch and M. Halik, Nanoscale, 2014, 6, 1302213027.

19 T. de Roo, J. Haase, J. Keller, C. Hinz, M. Schmid, D. V. Seletskiy, H. Cölfen, A. Leitenstorfer and S. Mecking, Adv. Funct. Mater., 2014, 24, 2714-2719.

20 L. Chua, J. Zaumseil, J. Chang and E. C. Ou, Nature, 2005, 434, 194-199.
21 A. Yokoyama, H. Suzuki, Y. Kubota, K. Ohuchi, H. Higashimura and T. Yokozawa, J. Am. Chem. Soc., 2007, 129, 7236-7237.

22 T. Yokozawa and Y. Ohta, Chem. Rev., 2016, 116, 19501968.

23 M. Halik, H. Klauk, U. Zschieschang, G. Schmid, C. Dehm, M. Schütz, S. Maisch, F. Effenberger, M. Brunnbauer and F. Stellacci, Nature, 2004, 431, 963-966.

24 T. Lenz, T. Schmaltz, M. Novak and M. Halik, Langmuir, 2012, 28, 13900-13904.

25 L. Zeininger, L. Portilla, M. Halik and A. Hirsch, Chem. Eur. J., 2016, 22, 13506-13512.

$26 \mathrm{~S} . \mathrm{H}$. Chen, A. C. Su, C. H. Su and S. A. Chen, Macromolecules, 2005, 38, 379-385.

27 S. Tavazzi, A. Borghesi, M. Gurioli, F. Meinardi, D. Riva, A. Sassella, R. Tubino and F. Garnier, Synth. Met., 2003, 138, 55-58.

28 M. A. Loi, E. da Como, F. Dinelli, M. Murgia, R. Zamboni, F. Biscarini and M. Muccini, Nat. Mater., 2004, 4, 81-85.

29 A. Khassanov, H.-G. Steinrück, T. Schmaltz, A. Magerl and M. Halik, Acc. Chem. Res., 2015, 48, 1901-1908.

30 M. Novak, T. Schmaltz, H. Faber and M. Halik, Appl. Phys. Lett., 2011, 98, 93302.

31 Y. Yuan, G. Giri, A. L. Ayzner, A. P. Zoombelt, S. C. B. Mannsfeld, J. Chen, D. Nordlund, M. F. Toney, J. Huang and Z. Bao, Nat. Commun., 2014, 5, 3005.

32 M. Burkhardt, A. Jedaa, M. Novak, A. Ebel, K. Voïtchovsky, F. Stellacci, A. Hirsch and M. Halik, Adv. Mater., 2010, 22, 2525-2528.

33 L. Portilla and M. Halik, ACS Appl. Mater. Interfaces, 2014, 6, 5977-5982.

34 A. Rumpel, M. Novak, J. Walter, B. Braunschweig, M. Halik and W. Peukert, Langmuir, 2011, 27, 1501615023.

35 H. Klauk, G. Schmid, W. Radlik, W. Weber, L. Zhou, C. D. Sheraw, J. A. Nichols and T. N. Jackson, Solid-State Electron., 2003, 47, 297-301. 